\title{
Identificando a origem de fontes tipográficas a partir de um catálogo de tipos: o repertório do Specimen de Tipos da Tipografia Hennies Irmãos
}

\section{Identifying the origin of typefaces from a typographic catalogue: the repertoire of the Hennies Brothers letterpress printing shop Type Specimen}

\author{
Jade Samara Piaia, Universidade de São Paulo
}

jadepiaia@usp.br

Priscila Lena Farias, Universidade de São Paulo

prifarias@usp.br

\section{Resumo}

Muitos tipos circularam em São Paulo desde a instalação da primeira oficina tipográfica, em 1827, mas são escassos os registros e estudos sobre seu design. Este artigo aborda o repertório tipográfico da Tipografia Hennies Irmãos, oficina fundada em 1891 por imigrantes alemães, apresenta os métodos adotados para identificar as fundidoras que originalmente produziram estas fontes, expõe e discute os resultados da investigação. O estudo foi realizado a partir do catálogo Specimen de Tipos, Vinhetas, etc., publicado pela empresa na primeira metade do século XX. Os resultados demonstraram que parte significativa das fontes tipográficas utilizadas pelos Hennies neste período tiveram como origem a Alemanha.

Palavras-chave: história do design, cultura da impressão, fontes tipográficas, Berthold, Funtimod.

\begin{abstract}
Many typefaces circulated in São Paulo since the installation of the first letterpress printing shop in 1827, but there are few records and studies on their design. This article tackles the typographic repertoire of the Hennies Brothers letterpress printing shop, founded in 1891 by German immigrants, presents the methods adopted to identify the foundries that originally produced these typefaces, announces and discusses the results of the investigation. The study was conducted from the Specimen de Tipos, Vinhetas, etc. catalogue, issued by the Hennies in the first half of the 20th century. The results demonstrate that a significant part of the typefaces in use by the Hennies in this period were of German origin.
\end{abstract}

Keywords: design history, print culture, typeface, Berthold, Funtimod. 


\section{Introdução}

Este artigo apresenta resultados de uma pesquisa sobre a memória gráfica paulistana que investigou a Tipografia Hennies Irmãos, revelando sua trajetória e produções impressas, bem como seu repertório tipográfico e as origens desse repertório. Fundada pelos imigrantes alemães Heinrich Hennies e Theodor Hennies, a oficina iniciou suas atividades em 1891 e permaneceu em funcionamento até 1992. Durante o centenário de atuação, a empresa adotou diferentes nomenclaturas ${ }^{1}$, pautadas por suas composições societárias, incluindo duas gerações familiares posteriores à dos fundadores. A empresa atuou em diversos logradouros da cidade de São Paulo, tendo como endereço principal a Rua Riachuelo no 14-16. Há registros de mais de 430 impressos assinados por esta oficina, que compreendem livros e periódicos publicados em sete idiomas. Além de atuarem como impressores, anunciavam ser também representantes e importadores de diversas fábricas europeias, sendo portanto possível imaginar que tenham importado boa parte das fontes de seu repertório. Divulgavam em anúncios (figura 1), em meados da década de 1910, serem os primeiros, no Brasil, a fabricar cartões fantasia em alto-relevo e aerografia.

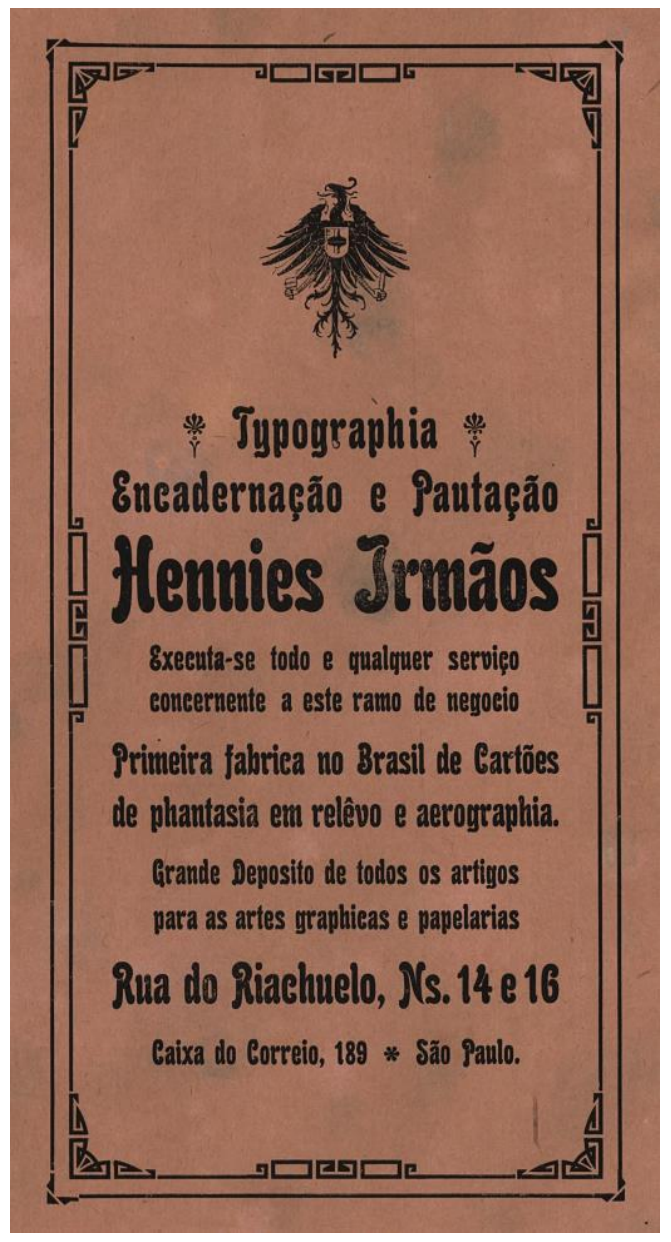

Figura 1: Anúncio da Tipografia Hennies Irmãos na revista Album Imperial, nº4, 1906.

Fonte: Arquivo Público do Estado de São Paulo.

\footnotetext{
${ }^{1}$ [1891] Hennies, Schulz e Cia. (Typ.); [1892] Hennies \& Winiger (Typ.); [1893-1925] Hennies \& Irmãos (Typ.); [1925-1934] - Hennies Irmãos \& Comp. / Hennies Irmãos \& Cia. (Typ.); [1934-1992] Hennies \& Cia. (Tipografia).
} 
O objetivo principal deste estudo foi estabelecer a origem dos desenhos das famílias de tipos móveis presentes no impresso Specimen de Tipos, Vinhetas, etc (HENNIES \& CIA., s.d.). Organizado como um catálogo, o objeto central desta investigação (figura 2) conta com 60 páginas medindo 150 × $225 \mathrm{~mm}$. Possui amostras de 354 fontes $^{2}$, organizadas em 86 famílias tipográficas ${ }^{3}$, considerando os diferentes pesos e corpos. Destas 354 fontes, 13 são tipos de madeira e 3 contêm somente números. O catálogo também contém 22 manículas (ornamentos em forma de mãos que apontam), 35 modelos de fios, 6 fundos e 5 emblemas para festas de natal e ano bom. Os tipos de madeira, números, fios, fundos e emblemas não foram abordados no estudo apresentado neste artigo.

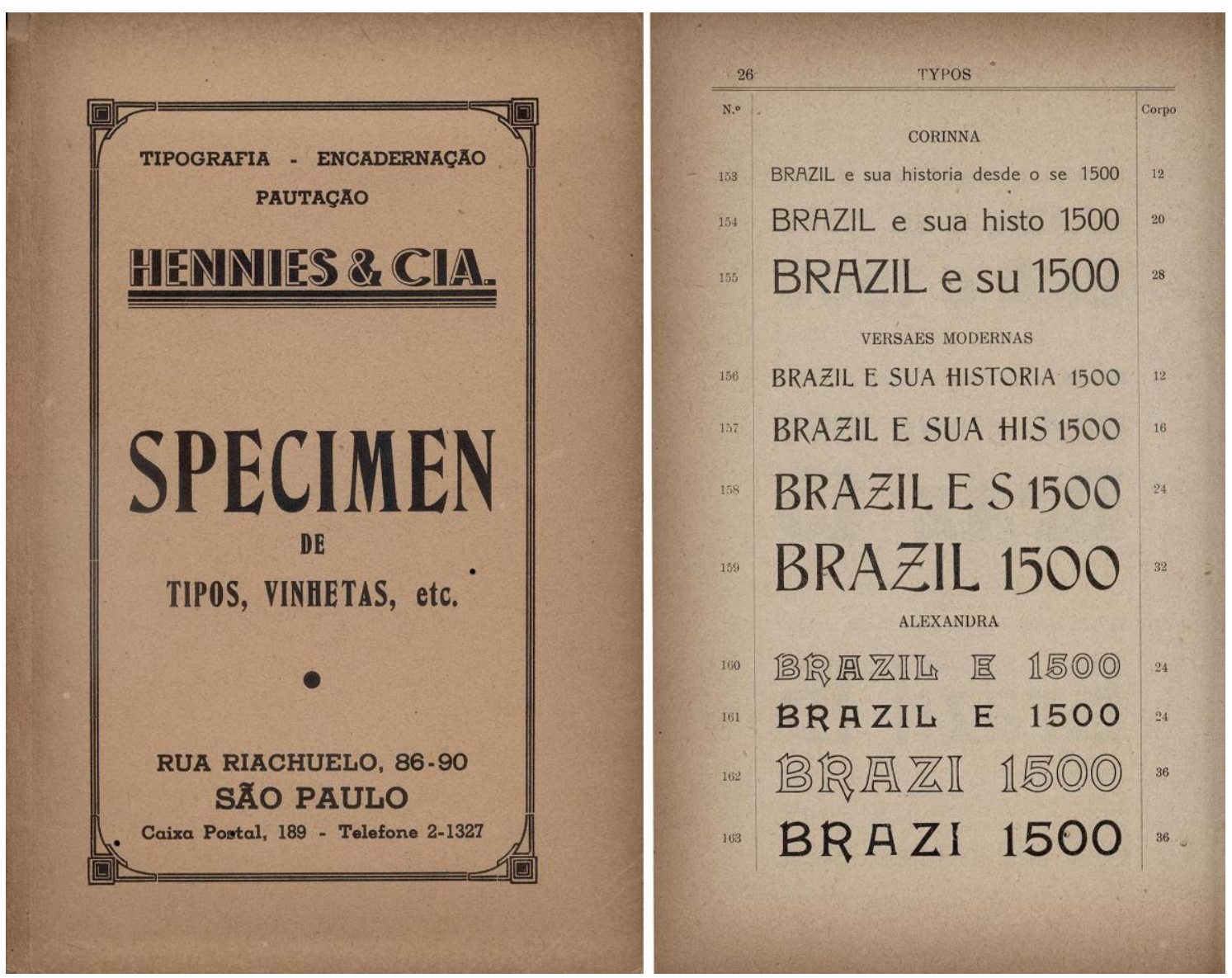

Figura 2: Capa e página interna do Specimen de Tipos, Vinhetas, etc. (HENNIES \& CIA., s.d.), acervo pessoal da Família Hennies.

A investigação almejou responder às seguintes questões: Os Hennies importavam fontes tipográficas exclusivamente da Alemanha? Quais eram os principais fornecedores dos tipos utilizados pela Tipografia Hennies Irmãos? Para respondê-las, partiu-se do pressuposto de que as fontes, ou ao menos uma parte delas, pudesse ter origem alemã, devido à nacionalidade dos irmãos Hennies. Este fato orientou os primeiros levantamentos, ainda exploratórios, em busca de fontes de pesquisa consistentes.

\footnotetext{
${ }^{2}$ Conjunto de caracteres com desenho e tamanho específicos.

${ }^{3}$ Conjuntos de caracteres com o mesmo desenho, em corpos diferentes.
} 
Um recorte foi necessário para iniciar as buscas, que envolveram principalmente materiais relacionados às fontes tipográficas que circulavam no final do século XIX e nas três primeiras décadas do século XX na Europa, sobretudo na Alemanha. A sistematização das buscas em um protocolo investigativo, dentro desse recorte, permitiu estabelecer uma sequência previsível de etapas para examinar uma amostra tipográfica (um conjunto de caracteres em determinado estilo). Com isso, cada amostra tipográfica encontrada no Specimen de Tipos, Vinhetas, etc (HENNIES \& CIA., s.d.), doravante denominado apenas como Specimen de Tipos, foi cuidadosamente checada, afunilando, a cada etapa, as possibilidades de busca por mais informações, até chegar em amostras de tipos similares àquelas utilizadas pelos Hennies, e que pudessem então ser comparadas mais atentamente. Partindo apenas dos nomes utilizados pelos Hennies para identificar as fontes (e também pesos e variações, em português) e de algumas amostras de caracteres, o protocolo funcionou como um filtro. À medida em que as etapas avançavam e algum resultado era obtido, surgiam novos direcionamentos, que apontavam para novas possibilidades de busca, seja o nome de uma (ou mais de uma) fundidora, o possível nome original da fonte ou família (ou a confirmação da nomenclatura utilizada pelos Hennies como original), para o nome do autor ${ }^{4}$ do desenho da fonte, ou mesmo o ano em que a fonte teria sido lançada no catálogo de determinada fundidora. $\mathrm{O}$ estudo se justifica pela carência de dados sobre as origens e influências do repertório tipográfico que circulou na cidade de São Paulo em um momento importante de estabelecimento e expansão das atividades gráficas.

Estudos que abordam a memória gráfica e tipográfica, como os de Farias, Aragão e Cunha Lima (2011) e Farias (et al., 2018), examinando amostras do repertório tipográfico que circulou principalmente no Rio de Janeiro e em São Paulo no século XIX e início do século XX, delinearam caminhos para pesquisas sobre as origens desse repertório. Outros estudos relevantes para a pesquisa aqui apresentada são aqueles voltados especialmente para espécimes de tipos. Entre estes, destaca-se Aragão (2010), que analisa os catálogos de tipos de duas fundidoras representativas do século XX no Brasil, a Funtimod e a Manig; Aragão, Farias e Cunha Lima (2014), que fazem uma análise comparativa entre catálogos da Fundição Franceza de E. Bouchaud \& Sobrinho, do Specimen da Fundição de Typos Henrique Rosa, publicados no Rio de Janeiro, e do catálogo da Funtymod, publicado em São Paulo; o trabalho de Cunha Lima, Aragão e Farias (2013) que descreve o conteúdo e a organização de quatro espécimes de tipos do acervo da Biblioteca Nacional, três deles publicados no Rio de Janeiro e um no Pará, entre o século XIX e início do século XX; e o método de identificação de tipos proposto por Aragão e Farias (2017) aplicado aos catálogos de tipos da Funtimod.

O método aqui descrito torna-se relevante em situações em que não se tem o conhecimento sobre a fundidora responsável pela manufatura de um conjunto de fontes tipográficas, tornandose pertinente a investigações de grande amplitude, nas quais há a necessidade de filtrar e reduzir as possibilidades de busca, otimizando o tempo empregado para chegar a informações concretas, e eventualmente a espécimes de tipos produzidos por fundidoras que possibilitem comparar amostras e confirmar suposições. Este artigo pretende abrir caminho para outras investigações

\footnotetext{
${ }^{4}$ Atualmente denominado designer de tipos, ou type designer.
} 
desta natureza, trazendo à luz importantes fontes de pesquisa compiladas em um protocolo investigativo, que sistematiza o afinamento de suposições até a checagem das amostras para confirmação.

Os resultados obtidos neste estudo confirmaram que a maioria das fontes utilizadas pelos Hennies tiveram como origem a Europa, especialmente a Alemanha. De 46 fontes tipográficas identificadas, verificou-se que 29 eram idênticas a fontes presentes no espécime da fundidora alemã H. Berthold (s.d.), além de dois conjuntos de ornamentos (contendo manículas), e 17 fontes presentes em catálogos da fundidora brasileira Funtimod. A quantidade de informações obtidas sobre parte das fontes pesquisadas revelam que a aplicação deste protocolo investigativo obteve sucesso dentro do recorte temporal e geográfico proposto.

\section{Métodos: o estabelecimento de um protocolo investigativo}

Para a criação do protocolo desenvolvido para esta pesquisa foi considerado como ponto de partida o método de identificação de tipos proposto por Aragão e Farias (2017). Este consiste em um comparativo entre as amostras de tipos, utilizado na investigação das origens dos tipos da Funtimod-Fundidora de Tipos Modernos-, empresa com sede em São Paulo e atuante no século XX, amplamente pesquisada por Aragão (2016).

Aplicar somente o método proposto por Aragão e Farias (2017) pareceu insuficiente para este estudo devido ao grande número de fundidoras que poderiam ser responsáveis pela manufatura das fontes tipográficas presentes no repertório examinado, impossibilitando iniciar a pesquisa por meio de comparações entre amostras tipográficas. Os tipos da Tipografia Hennies poderiam ter vindo de qualquer fundidora da Alemanha, da Europa ou do Brasil, não havendo qualquer tipo de informação a esse respeito no Specimen de Tipos (HENNIES \& CIA., s.d.). Não havia, antes de se iniciar o estudo, uma relação conhecida entre os Hennies e alguma fundidora, exceto a Funtimod. A compra de tipos desta fundidora foi relatada por Waldemar Hennies em entrevistas (Piaia, 2018; 2020), referindo-se a aquisições realizadas nas décadas de 1960 e 1970 (posteriores, portanto, à data estimada de impressão do Specimen de Tipos, na década de 1930).

Sete fontes de pesquisa e uma etapa comparativa foram organizadas em oito passos sequenciais que compõem o protocolo investigativo. Este protocolo, resumidamente, compreende: consultas a livros e fichas de catalogação de fontes tipográficas (etapas 1, 2 e 3); buscas por dados sobre fontes e designers de tipos em plataformas digitais (etapas 4 e 5); busca por espécimes de tipos contendo amostras similares (etapa 6); busca por informações complementares em outras plataformas online (etapa 7); e comparação de amostras (etapa 8). O método de identificação de tipos (ARAGÃO, FARIAS, 2017) foi aplicado na última etapa do protocolo aqui descrito, quando as amostras das fontes tipográficas utilizadas pelos irmãos 
Hennies foram comparadas com amostras de tipos produzidos pelas fundidoras $\mathrm{H}$. Berthold ${ }^{5} \mathrm{e}$ Funtimod.

As respectivas fontes de pesquisa, seus conteúdos e procedimentos de consulta, e a aplicação do comparativo são relatados a seguir.

\section{1) Consultas ao Handbuch der Schriftarten}

O Handbuch der Schriftarten é um "manual de fontes"6 publicado por Emil Wetzig em 1926. Nele, o autor compila informações sobre tipos originários de fundidoras alemãs. Posteriormente o volume teve seis atualizações publicadas (WETZIG, Nachtrag 1929, 1930, 1931-1932, 19331935, 1936-1937, 1938-1939) que foram disponibilizadas digitalmente pelo Klingspor Museum.

A publicação agrupa amostras de vários períodos em uma lista ordenada e prática para consulta, com indicação das fundidoras que concordaram em participar do manual e enviaram o conteúdo. Segundo o autor (WETZIG, 1926), muitas fundidoras teriam sido adquiridas por outros proprietários, ou por fundidoras maiores, e rebatizadas, dificultando a compreensão da origem de muitas das fontes listadas. Para complicar o quadro, algumas famílias tipográficas receberam nomes diferentes ao serem comercializadas por empresas diferentes. O Handbuch der Schriftarten inclui amostras de fontes comercialmente disponíveis, no momento de sua publicação, em fundições localizadas na Alemanha, Áustria e Suíça.

No Handbuch der Schriftarten, as fontes estão divididas em categorias que incluem: "Gotische und Halbgotische" (góticas e semi-góticas); "Schwabacher und Kanzleischriften" (bastardas e letras de chancelaria); "Fraktur" (fraturas); "Deutsche Schrägschriften" (letras inclinadas alemãs); "Neudeutsche Schriften" (novas letras alemãs); "Antiquaschriften mit Kursiv" (romanas com itálico); "Medievalenschriften mit Kursiv" (serifadas com itálico); "Renaissance, Etienne, Egyptienne" (renascentistas, com serifas triangulares, egipcianas); "Reklame und Zierschriften" (letras para propaganda e ornamentais); "Groteskschriften mit Kursiv" (grotescas com itálico); "Akzidenz-Srägschriften" (comerciais inclinadas); "Schreibschiften, Schreibmaschiften" (letras escriturais e de máquina de escrever); "Griechische, Cyrillische (Russische), Orientalische und andere" (letras gregas, cirílicas [russas], orientais e outras). O manual possui ainda um apêndice dedicado a fontes para linotipo e monotipo.

O Handbuch der Schriftarten possui um sumário em ordem alfabética que pode facilitar a busca a partir do nome, ou de parte do nome, de uma família tipográfica. Deve-se atentar para a necessidade de traduzir para o alemão as nomenclaturas de peso, caso façam parte do nome da família tipográfica, bem como para mudanças na grafia das nomenclaturas em alemão. Cada família tipográfica traz informações de nomenclatura, algumas contêm o ano de lançamento e o nome do autor do desenho, o nome da fundidora e a cidade de origem, os corpos disponíveis e

\footnotetext{
${ }^{5}$ Principal nome pelo qual ficou conhecida internacionalmente a junção das fundidoras H. Berthold AG. e Bauer \& Co.

6 Tradução das autoras.
} 
uma pequena amostra da fonte aplicada em uma palavra, geralmente em corpo 16 pontos (figura $3)$.

\section{Korinna \\ H. Berthold 1904 \\ H. Berthold A.-G., Berlin \\ $6,8,9,10,12,14,16,20,24,28,36,48,60,72$ auf 60 \\ Hamburgers}

Figura 3: Detalhe das informações e amostra da fonte Korina (WETZIG, 1926, p. 153).

\section{2) Consultas ao Internationales Verzeichnis der Bleisatz-Schriften}

O Internationales Verzeichnis der Bleisatz-Schriften, publicado em 2011, é um "Registro Internacional dos Tipos de Metal"7, compilado por Hans Reichardt e apoiado financeiramente pelas fundidoras de tipos alemãs H. Berthold AG, Linotype AG e URW. O diretório contém fontes da Europa, América, Ásia e Austrália. Seu conteúdo teve como base outra obras, como o Handbuch der Schriftarten (publicado por Wetzig em 1926, e as atualizações publicadas até 1939), The Encyclopaedia of Typefaces (livro publicado por Jaspert, Berry e Johnson em 1970) e American Metal Typefaces of the Twentieth Century (livro publicado por Mac McGrew em 1993). Reichardt (2011) reuniu o conteúdo dessas obras, complementou com amostras que ele mesmo coletou e incluiu dados obtidos em outras fontes de pesquisa. Cobre especialmente famílias tipográficas disponíveis como tipos móveis no século XX.

Contém o nome da fonte, ou da família, o nome do autor do desenho (quando conhecido), o ano de lançamento (ou estimativa) e a fundidora fabricante ou proprietária dos desenhos. Outros fornecedores do mesmo tipo de letra ou de imitações aparecem em itálico, abaixo do nome. No caso das imitações, o nome original da fonte aparece abaixo do nome, destacado em itálico meio preto. Amostras de tipos são fornecidas quando se trata de um desenho original.

O conteúdo foi organizado em ordem alfabética pelo nome das famílias tipográficas e uma série de digitalizações das páginas do livro, separadas por letra inicial, é disponibilizada no site do Klingspor Museum. O volume contém apêndices com lista de fabricantes de tipos organizada em ordem alfabética e por países, e lista dos designers de tipos ou autores dos desenhos das punções organizada pelo sobrenome em ordem alfabética, com dados de nascimento e falecimento (figura 4).

\footnotetext{
7 Tradução das autoras.
} 
ABCDEFGHIJKLMNOPQRSTUVWXYZ

abcdefghijklmnopqrstuvwxyz 1234567890

Augustea halbfett 1906 H. Berthold AG

ABCDEFGHIJKLMNOPQRSTUVWXYZ abcdefghijklmnopqrstuvwxyz 1234567890

ABCDEFGHIJKLMNOPQRSTUVWXYZ abcdefghijklimnopqrstuvwxyz 1234567890

ABCDEFGHIJKLMNOPQRSTUVWXYZ abcdefghijklmnopqrstuvwxyz 1234567890

Figura 4: Detalhe das informações e amostras da família tipográfica Augustea (REICHARDT, 2011, vol. A, p. 26).

3) Consultas ao Verein der Schriftgießereien - VdS Kartei Teil A-Z

O Verein der Schriftgießereien - VdS Kartei Teil A-Z (s.d.) engloba as fichas da Associação de Fundições de Tipos da Alemanha, fundada no início do século XX e composta pela maioria das empresas do ramo. A associação serviu para representar os interesses das fundidoras em questões econômicas, políticas e jurídicas.

As fichas, em boa parte preenchidas manualmente, contêm as fontes lançadas pelas fundidoras e uma amostra dos caracteres. As informações que trazem são o nome da família tipográfica, a data de registro, o nome da empresa e sua localização, a data de lançamento, a classificação e a data da carta enviada. Em um espaço abaixo dessas informações (ou no verso), amostras de caracteres da fonte foram coladas. Os modelos das amostras variam bastante e não trazem informações sobre o tamanho das fontes impressas (figura 5). A organização das fichas em ordem alfabética facilita a consulta ao material, que se encontra digitalizado e é disponibilizado pelo site do Klingspor Museum. 


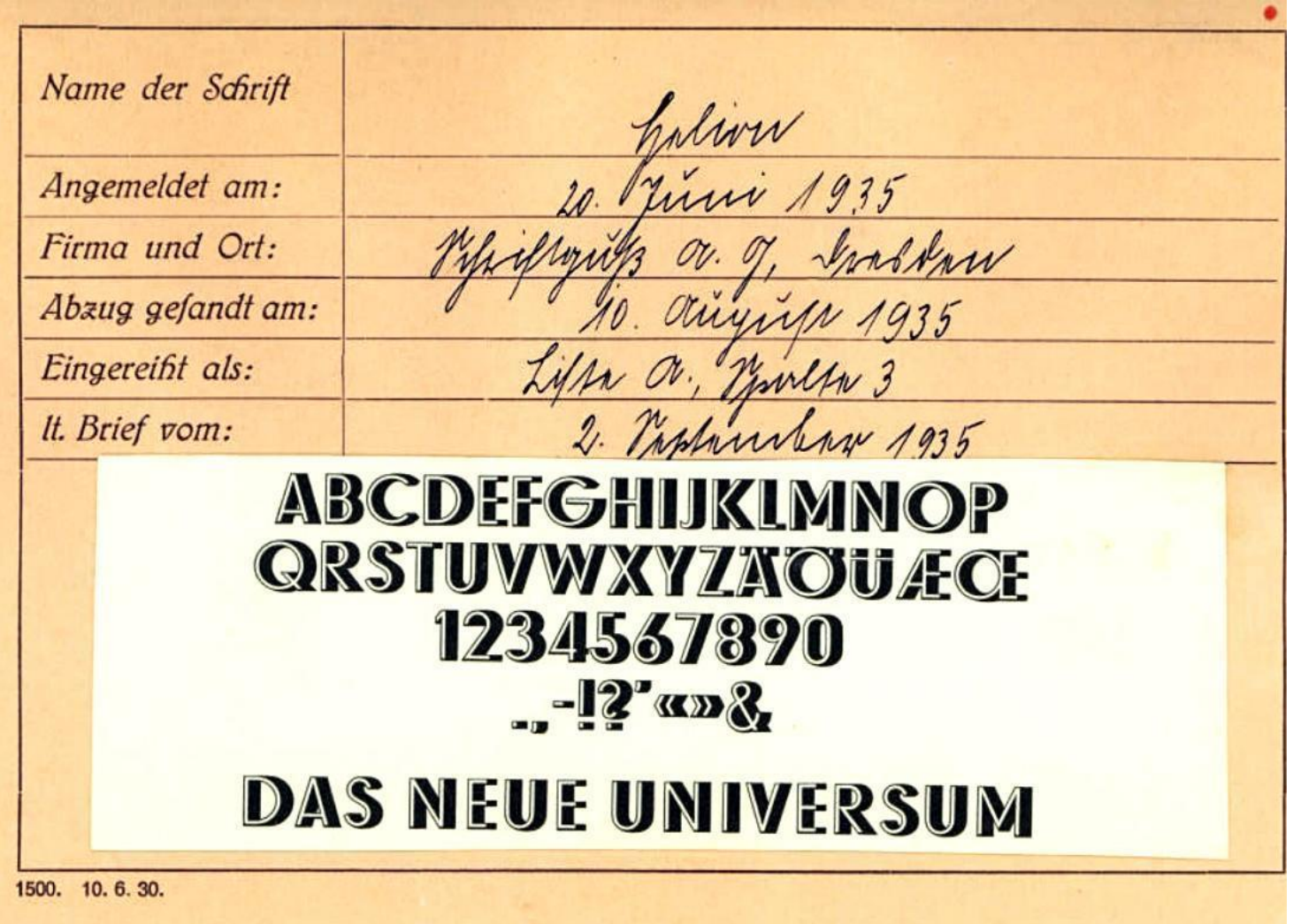

Figura 5: Ficha de registro da fonte Helion, 1935 (VEREIN DER SCHRIFTGIESSEREIEN, s.d.).

Esta fonte de dados cobre principalmente os tipos lançados na primeira metade do século XX na Alemanha. Os documentos da associação podem ser encontrados no Hessian Business Archive, em Darmstadt, e as fichas originais no Museu Klingspor, em Offenbach.

4) Consulta ao compêndio Typedesigner and Punchcutter: from Gutenberg until today

Typedesigner and Punchcutter: from Gutenberg until today é um volume digital (uma listagem, disponível em PDF) organizado por Hans Reichardt (2020), que reúne links para os mais de nove mil registros de designers de tipos registrados no site do Klingspor Museum. Está organizado em ordem alfabética pelo sobrenome. Contém o nome completo do designer de tipos, em alguns casos as datas e locais de nascimento e falecimento, e um link para outro arquivo em formato PDF com informações compiladas sobre o designer. No arquivo, além das informações pessoais e profissionais, constam informações e amostras das fontes desenhadas pelo designer. A quantidade de informação disponível sobre cada designer de tipos varia bastante, ocupando de meia a mais de 50 páginas.

\section{5) Consulta ao site de Luc Devroye}

O professor de ciência da computação e matemática na Universidade McGill, em Montreal, Luc Devroye, criou e mantém, desde 1992, um site com informações sobre tipografia e design de tipos contendo centenas de páginas sobre o assunto. 
Funcionando como uma enciclopédia, com acesso livre e gratuito, o site (LUC DEVROYE, s.d.) abrange dados históricos sobre fontes, fundidoras, designers de tipos e movimentos artísticos, entre outros aspectos. Frequentemente há amostras das fontes e exemplos de aplicações em projetos visuais. A busca na plataforma apresenta dados sobre fontes similares, cópias ou redesenhos, e links para versões digitais dos tipos, quando existentes.

A principal fontes dos dados compilados por Luc Devroye é o próprio Google, a Coleção de Livros Raros da Universidade McGill (com uma importante coleção de livros sobre tipografia e história da impressão no Canadá) e sites sobre atualidades no campo da tipografia como Typographica e MyFonts. Os resultados das buscas nesta plataforma podem ser bastante extensos, dependendo do termo de busca, e caberá ao pesquisador um cuidadoso filtro dos resultados.

\section{6) Busca por catálogos de tipos}

A busca por catálogos ou espécimes de tipos não costuma ser fácil, já que o acesso a esse tipo de conteúdo digitalizado é escasso devido a questões de catalogação e direitos autorais. Muitas vezes o único argumento de busca que se tem é o nome de uma família tipográfica, ou às vezes nem isso, sem referência exata de quais espécimes poderiam conter as amostras desejadas, e muitas vezes sem dados sobre fundidora e data de lançamento, torna-se necessário mergulhar em uma busca por pistas visuais e históricas, direcionadoras para a pesquisa.

Livros sobre catálogos de tipos são mais acessíveis do que os raros espécimes de tipos. Um possível caminho envolve a busca por referências em obras mais gerais sobre história do design que incluem informações sobre o design e uso de tipos, como Meggs \& Purvis (2009), e posteriormente em publicações mais específicas, como McNeil (2017), Heller \& Fili (1999) e Gray (1951). Bons exemplos de livros que reúnem reproduções de páginas de catálogos de tipos são os dois volumes de Type - A visual history of typefaces and graphic styles (JONG, PURVIS \& THOLENAAR, 2009; 2010).

As coleções físicas de espécimes de tipos estão espalhadas em museus e acervos de bibliotecas pelo mundo. Stephen Coles (2017) cita as principais coleções de livre acesso: na Alemanha: Gutenberg Museum (Mainz), Klingspor Museum (Offenbach), Letter Library (Weimar); na Bélgica: Plantin-Moretus (Antwerp); nos Estados Unidos: Columbia (New York), Lubalin Center (New York), Museum of Printing (Haverhill), Newberry (Chicago), Updike (Providence), U.S. Library of Congress (Washington D.C.); na França: Musée de l'Imprimerie (Lyon); na Holanda: University of Amsterdam; na Inglaterra: St. Bride (London), University of Reading; na Itália: Tipoteca (Treviso).

Espécimes digitalizados são relevantes por facilitar a consulta visual a uma grande quantidade de amostras em pouco tempo. São úteis independentemente do tamanho e da qualidade das imagens, desde que possibilitem um primeiro filtro por semelhança visual. As buscas por imagens digitalizadas de espécies de tipos realizadas para a pesquisa aqui relatada incluíram plataformas como Letter Library, Letterform Archive e Flickr. 
O Letter Library é um projeto do alemão Ralf Herrmann (s.d.) para a preservação da história da tipografia, que almeja a criação de um arquivo online com espécimes de tipos do mundo todo, digitalizados e de acesso livre. Está em andamento em colaboração com o museu da impressão Pavillon-Presse Weimar (s.d.). Este museu possui importantes coleções sobre tipografia de exprofessores como Schmidt e Jay Rutherford. Parte do conteúdo do Letterpress Library está presente e é atualizado constantemente nas redes sociais como Instagram, Facebook e Twitter. O Twitter facilita a busca por palavras-chave dentro do perfil (@Letter_Library) ${ }^{8}$ e exibe imagens maiores.

O Letterform Archive (s.d.), sediado em San Francisco na Califórnia, possui mais de 5.000 itens relacionados à tipografia e ao design de tipos. Recentemente compartilhou em seu site o acesso ao Online Archive, com parte de seu arquivo digitalizado. A categoria "type specimens" contava, na data da última consulta, com 268 itens que podiam ser filtrados por pessoa, empresa, década e país. As imagens têm excelente qualidade, mas não se trata necessariamente de digitalizações planas dos originais e as imagens não estão em tamanho real — foram redimensionadas para o site.

No Flickr 9 , uma comunidade de compartilhamento de imagens online, a busca utilizando filtros por palavras-chave como "type specimen" e "letterpress", associados ao nome da fonte pesquisada, bem como a pesquisa em grupos específicos relacionados ao assunto foram estratégias utilizadas para encontrar mais informações sobre o repertório da Hennies Irmãos. A busca frequentemente resulta em conteúdos inseridos por visitantes de museus e acervos que fotografaram as páginas com seus celulares.

Como parte da pesquisa relatada neste artigo, foi realizada consulta a um espécime de tipos da H. Berthold (s.d.), que inclui amostras de tipos modernos datados da virada do século XX. Este exemplar, de propriedade da família Hennies, é um forte indicativo da relação dos Hennies com esta fundidora de tipos alemã. $\mathrm{O}$ espécime foi digitalizado para facilitar a consulta e diminuir o desgaste do catálogo físico.

Foram também consultados catálogos da Funtimod, Fundidora de Tipos Modernos, empresa fundada em São Paulo em 1932, e que foi objeto de estudo de Aragão (2016). Considerada a maior fundidora de tipos do país, a empresa teve a colaboração dos alemães Karl H. Klingspor e Josef Tscherkassky na sua fundação e manteve relações com fundidoras alemãs como D. Stempel e Klingspor, das quais reproduziu tipos representativos do início do século XX. Dentre os dez catálogos de tipos da Funtimod, os cinco primeiros, produzidos entre as décadas de 1930 e 1960 (Catálogo I [1937?]; Catálogo II [entre 1937 e 1942]; Catálogo III [entre 1950 e 1956]; Catálogo IV [entre 1956 e 1958]; Catálogo V [entre 1958 e 1963]), foram consultados. Eles foram divulgados em período compatível com a publicação do Specimen de Tipos (HENNIES \& CIA., s.d.) e suas possíveis atualizações posteriores.

\footnotetext{
${ }^{8}$ Disponível em: <https://twitter.com/letter_library>. Acesso em: 19/06/2020.

${ }^{9}$ Disponível em: $<$ https://www.flickr.com/>. Acesso em: 19/06/2020.
} 


\section{7) Outras pesquisas online}

Em posse de alguma informação mais precisa sobre a amostra de tipos pesquisada é possível fazer algumas outras buscas na internet. No caso da pesquisa sobre o repertório tipográfico da Hennies Irmãos, a primeira delas incluiu uma busca no Google, utilizando o nome ou parte do nome da fonte no idioma original, nome da fundidora e do autor do desenho, todos os dados entre aspas. Isso frequentemente resultou na descoberta de outros acervos com imagens ou dados históricos. Quando o nome da fonte era significativamente particular foi possível ter algum retorno com o uso de hashtag a ele associada em redes sociais. Tais buscas, com resultados positivos mais raros, tiveram como resultados perfis de estudiosos e amantes da tipografia que eventualmente inseriram dados de acervos ou bancos de imagens, indicando assim outros caminhos para a investigação. Sites e blogs com discussão de conteúdo especificamente voltado à tipografia também foram consultados, tais como Typography Guru ${ }^{10}$ e Fonts In Use ${ }^{11}$.

É também possível fazer pesquisas por semelhança de imagens a partir das amostras de caracteres em plataformas que trabalham com o reconhecimento de fontes, como o WhatTheFont, do MyFonts ${ }^{12}$. Esse tipo de busca tem como resultado fontes digitais correspondentes e disponíveis na plataforma consultada. É interessante, neste caso, realizar a mesma consulta em diversas plataformas e, a partir da fonte digital semelhante encontrada, verificar o histórico de seu desenho, buscando informações sobre nomenclaturas antigas, fundidora de origem e autoria do desenho original. Resultados de buscas exclusivamente online tendem a ser mais expressivos para tipos produzidos no século XX.

\section{8) Comparação de amostras}

Os procedimentos relatados nos tópicos anteriores possibilitam comparações visuais apenas por observação, lado a lado, das amostras, o que leva a suposições que contribuem para filtrar possibilidades. Como último estágio de investigação, tendo em mãos imagens de amostras de caracteres em alta resolução (300dpi) e em tamanho real (preferencialmente obtidas por scanner plano), as famílias tipográficas presentes no Specimen de Tipos produzido pela Hennies Irmãos foram comparadas com imagens de fontes similares produzidas por diferentes fundidores, seguindo os procedimentos propostos por Aragão (2016). A comparação (figura 6) inicia com amostras que se supõe serem da mesma fonte por possuírem desenhos visualmente idênticos de caracteres, e tem por objetivo examinar a fundo os detalhes dos desenhos, confirmando que se trata da mesma fonte, ou de uma cópia ou versão alternativa com diferenças sutis.

\footnotetext{
${ }^{10}$ Disponível em: <https://typography.guru/>. Acesso em: 19/06/2020.

${ }^{11}$ Disponível em: <https://fontsinuse.com/>. Acesso em: 19/06/2020.

${ }^{12}$ Disponível em: $<$ https://www.myfonts.com/WhatTheFont/>. Acesso em: 19/06/2020.
} 


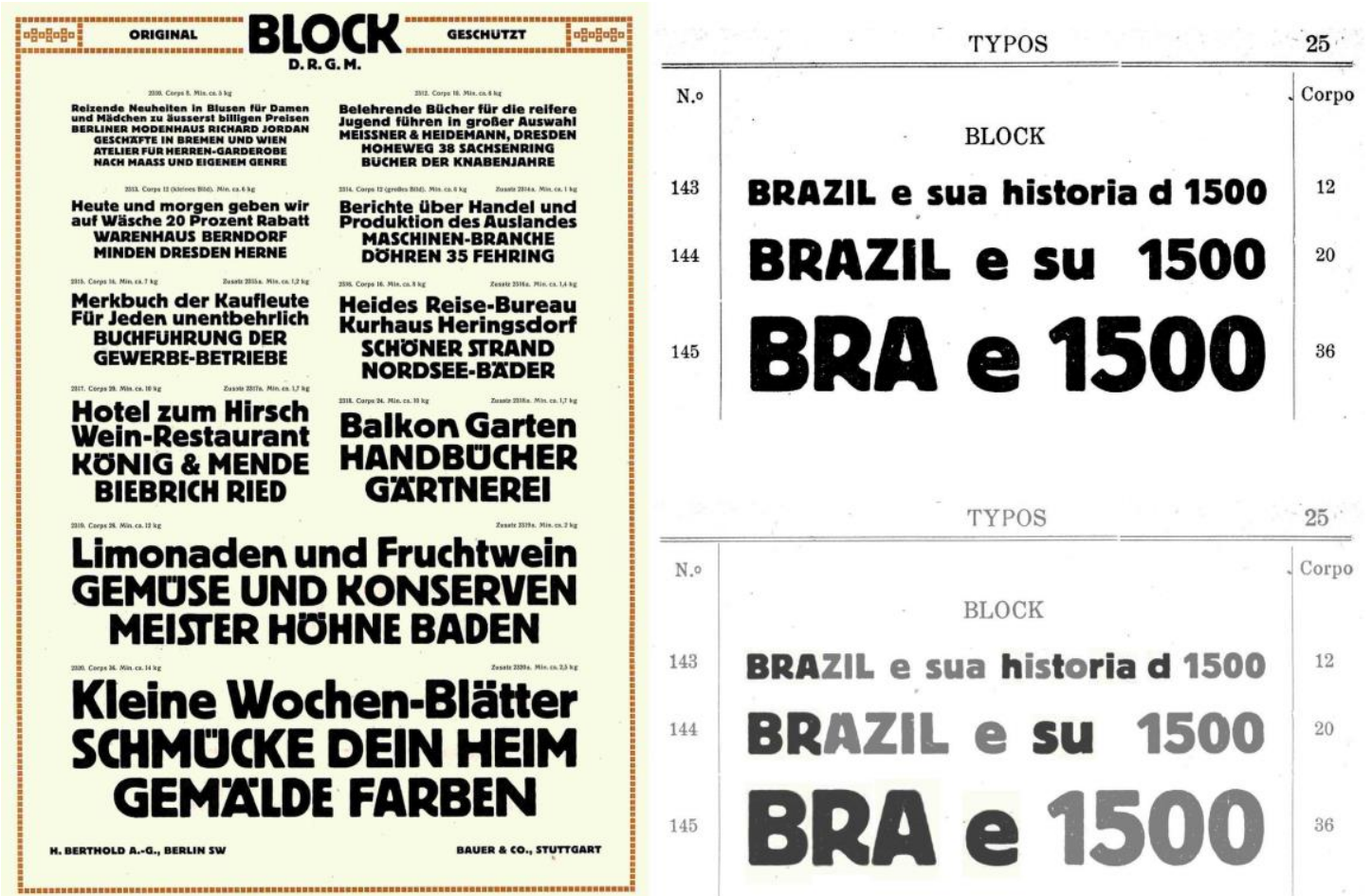

Figura 6: Amostras da fonte Block provenientes do espécime de tipos da H. Berthold, à esquerda, e do espécime da Hennies, acima à direita. $\mathrm{O}$ comparativo entre as amostras realizado por sobreposição das imagens, abaixo à direita, destaca os caracteres semelhantes. Imagem elaborada pelas autoras a partir de impressos do acervo pessoal da família Hennies.

Seguindo os procedimentos adotados por Aragão (2016), imagens de amostras de tipos das fundidoras H. Berthold (s.d.) e Funtimod (entre 1930 e 1960) foram sobrepostas, utilizando o recurso de camadas do software Adobe Photoshop, a imagens de amostras do Specimen de Tipos (HENNIES \& CIA., s.d.), para as quais os passos anteriores indicavam um alto grau de compatibilidade. Os resultados dessa etapa foram divididos em "confirmação satisfatória", para as fontes com $100 \%$ de compatibilidade entre as amostras e "não confirmados", para as fontes que apresentaram pequenas diferenças no desenho ou na espessura dos caracteres. As comparações foram realizadas apenas com as fontes para as quais foi possível obter imagens de amostras em tamanho original.

\section{Resultados}

Os resultados obtidos com os oito passos do protocolo investigativo foram organizados em planilhas. A organização partiu dos dados contidos no Specimen de Tipos da Hennies \& Cia. (s.d.): numeração e nomenclatura da fonte, página e corpo. Além dos oito campos para indicar se houve retorno nos passos do protocolo investigativo, a planilha compreende campos para o nome original da fonte, autor do desenho, ano, fundidora(s) identificada(s), cidade e país de origem, corpos existentes, classificação (originalmente atribuída na literatura consultada), fontes das informações e um campo para observações.

No total, das 86 famílias tipográficas presentes no Specimen de Tipos (HENNIES \& CIA., s.d.), dois conjuntos de ornamentos mais 46 famílias foram identificadas e confirmadas: 29 delas 
aparecem também no catálogo de tipos da fundidora alemã H. Berthold (s.d.), além dos dois conjuntos de ornamentos e 17 em catálogos da Funtimod publicados entre as décadas de 1930 e 1960 (figura 7).

\section{Identificação e confirmação satisfatória}

\begin{tabular}{|c|c|c|c|}
\hline Nomenclatura Hennies & Nome original & Ano & Fundidora \\
\hline Antiga Salão & Antiga Salão & 1906 & Funtimod \\
\hline Gladiola & Gladiola & 1936 & Funtimod \\
\hline Grotesca Reforma Gorda Apertada & Grotesca Reforma Gorda Apertada & - & Funtimod \\
\hline Grotesque Largo Magro & Grotesca Larga Clara & 1913 & Funtimod \\
\hline Grotesque Largo Meio Preto & Grotesca Larga Meia Preta & 1913 & Funtimod \\
\hline Grotesque Magro & Grotesca Reforma Magra & - & Funtimod \\
\hline Grotesque Meio Preto * & Grotesca Normal Meia Preta & - & Funtimod \\
\hline Grotesque Meio Preto & Halbbreite Grotesk & - & H. Berthold AG. \\
\hline Grotesque Meio Preto & Grotesca Normal Clara & - & Funtimod \\
\hline Kabel Claro ** & Kabel Magro Especial & após 1927 & Funtimod \\
\hline Kabel Claro ** & Kabel Magro & após 1927 & Funtimod \\
\hline Kabel Normal ** & Kabel Normal Especial & após 1930 & Funtimod \\
\hline Kabel Normal ** & Kabel Normal & após 1930 & Funtimod \\
\hline Kabel Meio Preto *** & Kabel Meio Preto Especial & após 1928 & Funtimod \\
\hline Mondial Magro *** & Mondial Magro & 1936 & Funtimod \\
\hline Typos de Maquina * & Escritura à Maquina & - & Funtimod \\
\hline Victoria & Vitória & - & Funtimod \\
\hline Visite & Visite & 1900-1901 [?] & Funtimod \\
\hline Alexandra & Zweifarbenschrift Alexandra & $1894[?]$ & H. Berthold AG. \\
\hline Augustea & Antiqua Augustea & 1905 & H. Berthold AG. \\
\hline Augustea Gripho & Augustea Cursiv & 1906-1907 [?] & H. Berthold AG. \\
\hline Augustea Meio Preto & Halbfette Augustea & 1906-1907 [?] & H. Berthold AG. \\
\hline Block & Block & 1908 & H. Berthold AG. \\
\hline Canon Angelus & Angelus Mediäval & antes de 1898 & H. Berthold AG. \\
\hline Corinna & Corinna & $1898[?]$ & H. Berthold AG. \\
\hline Egypcienne Versaes Preto & Schmale fette Egyptienne & $1870[?]$ & H. Berthold AG. \\
\hline Franklin * & Franklin & $1898[?]$ & H. Berthold AG. \\
\hline Grypho Grotesque Magro & Aviso-Cursiv & 1903 & H. Berthold AG. \\
\hline Grypho Grotesque Meio Preto & Halbfette Aviso & 1903 & H. Berthold AG. \\
\hline Hudson & Hudson & vor 1898 & H. Berthold AG. \\
\hline Imperial Manuscripto & Schribschrift Imperial & 1901 & H. Berthold AG. \\
\hline Imperial Manuscripto Meio Preto * & Halbfette Imperial & 1902 & H. Berthold AG. \\
\hline Iniciaes Brevier & Brevier-Initialen & $1898[?]$ & H. Berthold AG. \\
\hline Iniciaes Rococo & Rokoko-Initialen & antes de 1896 & H. Berthold AG. \\
\hline Latim & Lateinisch & 1899 & H. Berthold AG. \\
\hline Latim Gripho & Lateinisch-Cursiv & 1899 & H. Berthold AG. \\
\hline Latim Meio Preto & Halbfette Lateinisch & 1899 & H. Berthold AG. \\
\hline Latim Gripho Meio Preto & Halbfette Lateinisch-Cursiv & 1899 & H. Berthold AG. \\
\hline Loreley & Loreley & 1896 & H. Berthold AG. \\
\hline
\end{tabular}




\begin{tabular}{llll}
\hline Moderne Egyptienne & Moderne fette Egyptienne & 1898 [?] & H. Berthold AG \\
\hline Prinzess & Kartenschrift Princeß & 1905 & H. Berthold AG. \\
\hline Romana & Antique Schreibschrift Romana & 1898 & H. Berthold AG. \\
\hline Secession Meio Preto & Halbfette Secession & 1903 & H. Berthold AG. \\
\hline Secession & Secession & 1901 & H. Berthold AG \\
\hline Sorbonne Preto * & Fette Sorbonne & 1908 & H. Berthold AG. \\
\hline Versaes Modernas & Moderne Versalien & $1898[?]$ & H. Berthold AG. \\
\hline Mãos * **** & Hände & - & H. Berthold AG. \\
\hline Mãos **** & Neue Hände Serie 595 & - & H. Berthold AG.
\end{tabular}

*Há um ou mais corpos com comparação insatisfatória, ou há corpos de fontes similares no mesmo conjunto. **Há duas variações de fonte na amostra: Kabel Magro Especial e Kabel Magro; Kabel Normal Especial e Kabel Normal; o que pode indicar que possuíam as 2 fontes e houve uma mistura na composição. ***Ao menos um corpo corresponde a amostras em catálogos diferentes da mesma fundidora. **** Conjuntos de ornamentos (manículas).

Figura 7: Fontes tipográficas e conjuntos de ornamentos (manículas) que tiveram suas origens identificadas e confirmadas. Fonte: elaborado pelas autoras.

Seis famílias tipográficas foram identificadas como possivelmente provenientes da fundidora H. Berthold mas tiveram confirmação insatisfatória (figura 8). Elas possuem semelhanças na maior parte do desenho mas apresentam diferenças pontuais como letras únicas, diferenças de espessura, traços finos com falhas e/ou excesso de tinta em algumas áreas. Algumas ocorrências levantam a suspeita de tipos quebrados, outras de tipos desgastados e entupidos, sem descartar a possibilidade de serem cópias desses tipos obtidos de outras fundidoras.

\begin{tabular}{llll}
\hline \multicolumn{4}{c}{ Identificação com confirmação insatisfatória } \\
\hline Nomenclatura Hennies & Nome original & Ano & Fundidora \\
\hline Garmond Mediaeval & Mediaeval-Schriften & - & H. Berthold AG. \\
\hline Garmond Mediaeval & Mediaeval-Cursiv & - & H. Berthold AG. \\
\hline Mediaeval & Mediaeval-Schriften & - & H. Berthold AG. \\
\hline Mediaeval Estreito & Schmale Mediaeval & - & H. Berthold AG. \\
\hline Mediaeval Grypho & Mediaeval-Cursiv & - & H. Berthold AG. \\
\hline Sorbonne Circular & Sorbonne Circular & $1905 / 1906$ & H. Berthold AG. \\
\hline
\end{tabular}

Figura 8: Fontes tipográficas que tiveram suas possíveis origens identificadas mas com a confirmação insatisfatória.

Fonte: elaborado pelas autoras.

Vinte e seis famílias tipográficas foram identificadas como possivelmente originárias de 14 fundidoras, uma italiana e 13 alemãs (figura 9), mas a confirmação dessas identificações ainda depende de comparações com amostras em alta resolução extraídas de espécimes de tipos.

\section{Identificação a confirmar}

\begin{tabular}{llll}
\hline Nomenclatura Hennies & Nome original & Ano & Fundidora \\
\hline Hansa Grypho & Hansa-Kursiv & ca. 1895 & A.G. für Schriftgiess u. M. \\
\hline Iniciaes Circular Italiennes & Zirkular-Italienne & após 1891 & Baur'schen Buchdruckerei \\
\hline
\end{tabular}




\begin{tabular}{|c|c|c|c|}
\hline Renaissance Estreito & Renaissance Schmale & 1909 & $\begin{array}{l}\text { Bauersche Giesserei / } \\
\text { Genzsch \& Heyse / } \\
\text { Haas'sche Schriftgiesserei / } \\
\text { J. John Söhne / } \\
\text { Gebr. Klingspor }\end{array}$ \\
\hline Künstler-Schrift Claro & Künstlerschrift & 1902 & D. Stempel A.G. \\
\hline Künstler-Schrift Meio Preto & Künstlerschrift Halbfett & 1901 & D. Stempel A.G. \\
\hline Memphis Claro & $\begin{array}{l}\text { Memphis Zart / } \\
\text { Magere Memphis }\end{array}$ & $1930-1932[?]$ & D. Stempel A.G. \\
\hline Memphis Meio Preto & Halbfette Memphis & 1929 & D. Stempel A.G. \\
\hline Gloria & Schmale fette Gloria & antes de 1901 & Emil Gursch \\
\hline Elzevir & Elzevir 1 & 1899-1901 [?] & $\begin{array}{l}\text { Emil Gursch / } \\
\text { H. Berthold AG. / } \\
\text { Bauersche Giesserei }\end{array}$ \\
\hline Elzevir Meio Preto & Elzevir Halbfette & 1901 & $\begin{array}{l}\text { Emil Gursch / } \\
\text { Bauersche Giesserei }\end{array}$ \\
\hline Elzevir Gripho & Elzevir 1 Kursiv & 1899-1901 [?] & $\begin{array}{l}\text { Emil Gursch / } \\
\text { H. Berthold AG. }\end{array}$ \\
\hline Elzevir Estreito Preto & Elzevir Schmale Halbfette & $1899[?]$ & $\begin{array}{l}\text { Emil Gursch / } \\
\text { H. Berthold AG. }\end{array}$ \\
\hline Felicia & Felicia & 1911 & H. Berthold AG. \\
\hline $\begin{array}{l}\text { Garmond Mediaeval } \\
\text { [gripho meio preto] }\end{array}$ & Mediäval-Kursiv, Halbfette & - & H. Berthold AG. \\
\hline Mediaeval Grypho Meio Preto & Mediäval-Kursiv, Halbfette & - & H. Berthold AG. \\
\hline Circular Italienne & Zirkular Italienne & 1891 & J. G. Schelter \& Giesecke \\
\hline Iniciaes Gothicas & $\begin{array}{l}\text { Initialen / } \\
\text { Gotiche Fregiate - serie } 631\end{array}$ & $1911[?]$ & $\begin{array}{l}\text { J. G. Schelter \& Giesecke / } \\
\text { Nebiolo }\end{array}$ \\
\hline Schäffer Versalien & Radium & 1904-1905 [?] & $\begin{array}{l}\text { J. G. Schelter \& Giesecke / } \\
\text { Ludwig \& Mayer }\end{array}$ \\
\hline Schäffer Versalien & Schaefer Versalien & 1927 & $\begin{array}{l}\text { Schriftguss A.G. vorm. } \\
\text { Brüder Butter }\end{array}$ \\
\hline Butterfly Claro & Butterfly & 1927 & $\begin{array}{l}\text { Schriftguss A.G. vorm. } \\
\text { Brüder Butter }\end{array}$ \\
\hline Butterfly Meio Preto & Butterfly, Halbfette & 1928 & $\begin{array}{l}\text { Schriftguss A.G. vorm. } \\
\text { Brüder Butter }\end{array}$ \\
\hline Hollandeza Preta & Holländisch fette & 1925 & $\begin{array}{l}\text { Schriftguss A.G. vorm. } \\
\text { Brüder Butter }\end{array}$ \\
\hline Helion & Helion & 1935 & $\begin{array}{l}\text { Schriftguss A.G. vorm. } \\
\text { Brüder Butter }\end{array}$ \\
\hline Etrusco & Etrusco Nera Strettissima & $1920[?]$ & Società Nebiolo \\
\hline Veltro & Veltro & 1931-1934 [?] & Società Nebiolo / Linotype \\
\hline Veltro & Veltro Nera & 1934 & Società Nebiolo / Linotype \\
\hline
\end{tabular}

Figura 9: Famílias tipográficas com identificação a confirmar. Fonte: elaborado pelas autoras.

Somente oito famílias tipográficas, que possuem nomenclatura genérica, não foram identificadas: Grotesque Estreito (quatro pesos ${ }^{13}$ ), Romano (dois pesos ${ }^{14}$ ), e Typos de Machina

${ }^{13}$ Grotesque Estreito, Grotesque Estreito Meio Preto, Grotesque Meio Estreito, Grotesque Meio Estreito Preto.

${ }^{14}$ Romano, Romano Meio Preto. 
para Escrever (duas variações ${ }^{15}$ ). As versais das fontes Augustea, Elzevir, Mediaeval e Latim não constam no espécime da H. Berthold (s.d.) e por isso não foram comparadas nem confirmadas.

\section{Discussão}

Ao final da análise foi possível estimar que o Specimen de Tipos (HENNIES \& CIA., s.d.) tenha sido impresso após o ano de 1935, devido à presença da família tipográfica Helion (p. 28), lançada em junho de 1935 (VEREIN DER SCHRIFTGIESSEREIEN, s.d.). Após a primeira impressão o espécime parece ter sofrido ao menos duas alterações. Uma pode ser observada pelo papel mais claro (que manteve o aspecto branco preservado ao longo do tempo) com numerações que envolvem números e letras, como a página 24-A e 24-B, inserindo novas amostras próximas a letras no mesmo estilo; a outra alteração aparece no cabeçalho, com a atualização da grafia da palavra "Typos" para "Tipos"16, como nas páginas 32-A e 32-B.

Segundo depoimento do Sr. Waldemar Hennies (2020) as famílias tipográficas utilizadas nas folhas mais claras do Specimen de Tipos (HENNIES \& CIA., s.d.) foram compradas da Funtimod e o restante teria sido anteriormente importado da Alemanha por seus antecessores. $\mathrm{O}$ fato da família ter preservado o espécime da $\mathrm{H}$. Berthold (s.d.) se configura como um indicativo da origem de parte dos tipos. As datas de lançamento e o desenho das famílias tipográficas identificadas no Specimen de Tipos (HENNIES \& CIA., s.d.) reforçam que esse repertório abarca principalmente os tipos modernos do século XX. O Specimen de Tipos (HENNIES \& CIA., s.d.) não inclui tipos góticos ${ }^{17}$ (blackletter), tradicionalmente utilizados em impressos na Alemanha do século XIX, mas inclui duas famílias desse tipo criadas no século XX: Felicia, atribuída a Ferdinand Theinhardt, lançada em 1911, e Prinzess (Kartenschrift Princeß / Prinzess Kupferstichschrift), lançada em 1905 (WETZIG, 1926) - ambas encontradas também nas amostras do catálogo da H. Berthold (s.d.).

Com relação às famílias tipográficas da Funtimod, sabe-se, por Aragão (2016), que os desenhos de boa parte delas têm origem alemã, correspondendo àqueles de famílias tipográficas produzidas principalmente pelas fundidoras D. Stempel e Klingspor. Não é possível descartar a possibilidade de algumas das famílias tipográficas presentes no repertório da Typographia Hennies Irmãos terem sido importadas diretamente de fornecedores na Alemanha anteriormente ao início das atividades da Funtimod, em 1932 (ARAGÃO, 2016).

Não se descarta a possibilidade de terem existido outros espécimes de tipos anteriores a este (HENNIES \& CIA., s.d.). Ao examinar os impressos produzidos pelos Hennies, principalmente da primeira e parte da segunda décadas de atividade, há um conjunto significativo de fontes tipográficas utilizadas que não constam neste catálogo e merecerão um estudo futuro.

\footnotetext{
15 240, 240-B.

${ }^{16}$ Esta mudança foi prevista na reforma ortográfica de 1943 , portanto tais adendos devem ser posteriores a essa data.

17 Tradicionalmente utilizados na escrita alemã no início do século XX.
} 
Os procedimentos de pesquisa aqui relatados tiveram como principal argumento a busca pelo nome da fonte ou família tipográfica, preservado no caso do espécime da Hennies. Isso parece ser algo comum em situações em que as fontes eram importadas, principalmente da Europa para as Américas, e revendidas por outras empresas ou mesmo copiadas. A manutenção de nomes, porém, não aconteceu em outros espécimes de tipos que circularam no Rio de Janeiro no século XX, como o da Casa Granado ${ }^{18}$, no qual as famílias tipográficas foram identificadas por números sequenciais.

\section{Considerações finais}

Pouco se sabe sobre as origens das fontes tipográficas que circularam por São Paulo no período áureo da impressão com tipos móveis, iniciado na capital em 1827, e substituído à medida em que a linotipia e a composição a frio ganhavam o mercado gráfico, a partir de meados do século XX. Os estudos mais profundos que se tem até o momento, considerando o recorte geográfico em São Paulo, provém das pesquisas de Aragão $(2010 ; 2016)$ que revelam alguns dados sobre a fundidora Manig e, de forma mais detalhada, descrevem a trajetória e o repertório da fundidora Funtimod.

A formulação de um protocolo facilitou o desenvolvimento da investigação e garantiu sistematicidade na checagem de dados, permitindo atingir o objetivo de auxiliar no rastreamento e na identificação das origens das famílias tipográficas pertencentes ao repertório da Tipografia Hennies, e acredita-se que poderá auxiliar estudos similares.

Apesar dos resultados revelarem uma grande quantidade de famílias tipográficas e ornamentos provenientes da $\mathrm{H}$. Berthold, algumas delas, ao que tudo indica, podem ter sido adquiridas localmente da Funtimod. Outros possíveis fornecedores de tipos identificados, mas ainda não confirmados, incluem outras 12 fundidoras alemãs - A.G. für Schriftgiess u.M., Schriftguss A.G. vorm. Brüder Butter, Baur'schen Buchdruckerei, Bauersche Giesserei, D. Stempel, Emil Gursch, Gebr. Klingspor, Genzsch \& Heyse, Haas'sche Schriftgiesserei, J. G. Schelter \& Giesecke, J. John Söhne, Ludwig \& Mayer - e a fundidora italiana Società Nebiolo. Uma limitação observada neste estudo, no caso das fontes identificadas como provenientes dessas fundidoras, é que não foi possível aplicar a última etapa do protocolo, a comparação, por não ter sido possível ter acesso a catálogos de tipos produzidos por elas.

Em relação às questões norteadoras deste artigo, confirmou-se que os Hennies não importavam fontes tipográficas exclusivamente da Alemanha, mas pode-se dizer que seu portfólio de tipos era caracterizado majoritariamente por fontes com design alemão, tendo sido importadas ou compradas localmente, com a exceção das fontes provenientes da Nebiolo. Os Hennies se mantiveram ativos por pouco mais de um século e as fontes aqui investigadas cobrem os tipos que aparecem nas publicações principalmente a partir da segunda década de existência da empresa, após a virada do século XIX para o XX. A relação dos Hennies com o país de origem, a importação de tipos e suprimentos gráficos sofreram mudanças durante esses anos, pautadas

18 Acervo particular da empresa Granado, Rio de Janeiro. 
tanto pela disponibilização local dos tipos com design alemão através da Funtimod, quanto por mudanças na gestão da empresa, passada aos poucos para as gerações dos Hennies nascidos no Brasil.

A origem predominantemente alemã das fontes no repertório tipográfico dos Hennies confirma o compartilhamento de aspectos da cultura impressa por meio dos imigrantes em um momento de formação do campo gráfico paulistano. Acredita-se que, assim como neste caso, possam existir, em coleções particulares ainda não identificadas, outros espécimes de tipos e impressos efêmeros altamente relevantes para a caracterização da memória gráfica paulistana.

Revelar as principais fontes de pesquisa utilizadas neste artigo pode auxiliar outros pesquisadores, pois boa parte das fontes de pesquisa está acessível online. Estudiosos interessados em traçar este caminho reverso dos tipos móveis que cruzaram o oceano podem trazer à tona novos espécimes de tipos de fundidoras e revelar outros importadores e fundidoras que atuaram no país. No sentido metodológico, estudos futuros podem iluminar outras fontes de busca por espécimes de tipos acessíveis online e incluir procedimentos de identificação das fontes tipográficas envolvendo tecnologias de reconhecimento óptico de padrões, em constante aprimoramento.

\section{Agradecimentos}

Esta pesquisa foi apoiada por bolsas de Pós-Doutorado (FAPESP, n 2019/07566-6), e de Produtividade em Pesquisa (CNPQ, nº 304361/2019-4). Registramos os agradecimentos à família Hennies e à pesquisadora Isabella Aragão.

\section{Referências}

ARAGÃO, Isabella. Um breve panorama dos catálogos de tipos das fundidoras Funtimod e Manig. In: $9^{\circ}$ Congresso Brasileiro de Pesquisa e Desenvolvimento em Design 2010, 2010, São Paulo. Anais do 9 Congresso Brasileiro de Pesquisa e Desenvolvimento em Design 2010. São Paulo: PPG em Design, Universidade Anhembi Morumbi, AEND-Brasil, 2010.

ARAGÃO, Isabella. Tipos móveis de metal da Funtimod: contribuições para a história tipográfica brasileira. 2016. 282p. Tese de Doutorado. Universidade de São Paulo, São Paulo, 2016.

ARAGÃO, Isabella; FARIAS, Priscila; CUNHA LIMA, Edna. Um estudo sobre catálogos de tipos de fundidoras brasileiras dos séculos 19 e 20. In: COUTINHO, Solange et al. (org.). Anais do $6^{\circ}$ Congresso Internacional de Design da Informação, $5^{\circ}$ InfoDesign Brasil, $6^{\circ}$ CONGIC. São Paulo: Blucher Design Proceedings, n.2, v.1, 2014.

ARAGÃO, Isabella; FARIAS, Priscila. Identificando tipos móveis: metodologia para o estabelecimento da origem do design das faces tipográficas da Funtimod. In: Estudos em Design. Revista (online). Rio de Janeiro, v. 25, n. 2, p. 122-144, 2017. Semestral.

COLES, Stephen. Pre-digital Type Specimens: Aggregation, Examination, and Dissemination. Montréal: ATypI, Association Typographique Internationale, 2017. Disponível em: <https://www.youtube.com/watch?v=Z-JTUp8fXuM>. Acesso em: 17, jun. 2020. 
CUNHA LIMA, Edna; ARAGÃO, Isabella; FARIAS, Priscila. Describing movable type specimens: a contribution to Brazilian (tipo)graphic memory. In: Fadel, L. et al. (org.). Selected Readings of the 5th Information Design International Conference: research and practice. Florianópolis: SBDI, p. 233-249. 2013.

FARIAS, Priscila; ARAGÃO, Isabella; CUNHA LIMA, Edna. Unraveling aspects of Brazilian design history through the study of 19th century almanacs and type specimens. In: DRS 2012 Bangkok. Bangkok, Thailand: Chulalongkorn University, 2011.

FARIAS, Priscila L. et al. Designing the early history of typography in Brazil, starting from printing in São Paulo. In: Back to the Future. The Future in the Past [Proceeding Book Type and Histories: Past and Present Issues of Type and Book Design]: ICDHS 10th + I Conference. Barcelona, ICDHS 10th + I, 2018.

FUNTYMOD. Fundição de typos modernos Ltda. (Catálogo I). São Paulo: Funtymod, [1937?]. Fonte: acervo pessoal de Isabella R. Aragão.

(Catálogo II). São Paulo, Funtymod, [entre 1937 e 1942]. Fonte: acervo pessoal de Isabella R. Aragão.

FUNTIMOD. Fundição de tipos modernos S. A. (Catálogo III). São Paulo, Funtimod, [entre 1950 e 1956]. Fonte: acervo pessoal de Isabella R. Aragão.

(Catálogo IV). São Paulo, Funtimod, [entre 1956 e 1958]. Fonte: acervo pessoal de Isabella R. Aragão.

FUNTIMOD S. A. Máquinas e materiais gráficos. (Catálogo V). São Paulo, Funtimod S. A., [entre 1958 e 1963]. Fonte: acervo pessoal de Isabella R. Aragão.

GRAY, Nicolette. XIXth century ornamented types and title pages. Londres: Faber and Faber, 1951, 213 p.

H. BERTHOLD, Messinglinienfabrik und Schriftgiesserei A. G. Schriften und Ornamente: Messing-Universalblatt und Vignetten. Spezial-Kataloge: Über messing-erzeugnisse, sowie russische, griechische und orientalische schriften stehen interessenten zu diensten. Berlin SW, (sem data). Acervo: Família Hennies.

HELLER, Steven; FILI, Louise. Typology: Type Design from the Victorian Era to the Digital Age. San Francisco: Chronicle Books, 1999. 196p.

HENNIES IRMÃOS \& CIA. Catálogo. São Paulo: Tipografia, Encadernação, Pautação, Hennies Irmãos \& Cia, 1930. Acervo: Família Hennies.

HENNIES \& CIA. Specimen de tipos, vinhetas, etc. São Paulo: Tipografia, Encadernação, Pautação, Hennies \& Cia, (sem data). Acervo: Família Hennies.

HERRMANN, Ralf. Letter Library: the online archive of type specimens. Weimar, (sem data). Disponível em: <https://schriftkontor.ralf-herrmann.de/letterlibrary/>. Acesso em: 03, jun. 2020.

LETTERFORM ARCHIVE. Online Archive. San Francisco, (sem data). Disponível em: $<$ http://oa.letterformarchive.org/search?dims=Format\&vals0=Type\%20Specimen>. Acesso em: 03, jun. 2020.

LUC DEVROYE. School of Computer Science, McGill University: Montreal, Canada, (sem data). Disponível em: <http://luc.devroye.org/>. Acesso em: 03, jun. 2020.

MCNEIL, Paul. The Visual History of Type. Londres: Laurence King Publishing, 2017, 672p.

MCGREW, Mac. American Metal Typefaces of the Twentieth Century. Newcastle: Oak Knoll Books, 1993, 376p. 
MEGGS, Philip; PURVIS, Alston. História do Design Gráfico. São Paulo: Cosac Naify, 2009, 720p.

PAVILLON-PRESSE WEIMAR, Printing Museum. The Library. Weimar, (sem data). Disponível em: <https://pavillon-presse.de/en/the-library/>. Acesso em: 03, jun. 2020.

PIAIA, Jade S. Sr. Waldemar Hennies em 20/11/2018, domingo à tarde. Entrevista. Campinas, São Paulo, 2018. Transcrição não publicada.

PIAIA, Jade S. Sr. Waldemar Hennies em 20/02/2020, sábado à tarde. Entrevista. Campinas, São Paulo, 2020. Transcrição não publicada.

REICHARDT, Hans. [compilado por]. Internationales Verzeichnis der Bleisatz-Schriften / International Index of Hot-Metal Typefaces. Frankfurt am Main: Klingspor Museum, 2011. Disponível em: <http://www.klingspormuseum.de/KlingsporKuenstler/HR_Schriftbuch/HR_01_Titel-Vorwort.pdf $>$. Acesso em: 03, jun. 2020.

REICHARDT, Hans. Typedesigner and Punchcutter from Gutenberg until today I Schriftkünstler und Stempelschneider von Gutenberg bis zur Gegenwart. Frankfurt am Main: Klingspor Museum, 2020. Disponível em: <http://www.klingspormuseum.de/KlingsporKuenstler/Schriftdesigner/TypeDesigner.pdf $>$. Acesso em: 03, jun. 2020.

JONG, Cees W. de; PURVIS, Alston W.; THOLENAAR, Jan. Type, A Visual History of Typefaces and Graphic Styles. Vol. 1. Köln: Taschen, 2009. 360p.

JONG, Cees W. de; PURVIS, Alston W.; THOLENAAR, Jan. Type, A Visual History of Typefaces and Graphic Styles. Vol. 2. Köln: Taschen, 2010. 360p.

VEREIN DER SCHRIFTGIESSEREIEN. VdS Kartei Teil A-Z. Frankfurt am Main: Klingspor Museum, (sem data). Disponível em: <http://www.klingspor-museum.de/Vereinder-Schriftgiessereien-VdS.html>. Acesso em: 03, jun. 2020.

WETZIG, Emil. Handbuch der Schriftarten. Leipzig, Albrecht Seemann Verlag, 1926, 296p. Nachträge 1927, 1929, 1930, 1933/1935, 1936/1937, und 1938/1939. Disponível em: <http://www.klingspor-museum.de/Handbuch-der-Schriftarten.html>. Acesso em: 02, dez. 2019.

\section{Sobre as autoras}

\section{Jade Samara Piaia}

Pesquisadora de pós-doutorado no Laboratório de Pesquisa em Design Visual (LabVisual), Faculdade de Arquitetura e Urbanismo da Universidade de São Paulo (FAU USP). É doutora em Artes Visuais pelo Instituto de Arte da Universidade de Campinas (Unicamp). Tem experiência prática e docente na área de design gráfico. Seus interesses atuais de pesquisa são tipografia, memória gráfica, história do design, design da informação e comunicação visual.

ORCID < https://orcid.org/0000-0003-0191-5141 >.

\section{Priscila Lena Farias}

Professora Associada da Faculdade de Arquitetura e Urbanismo da Universidade de São Paulo (FAU USP), Coordenadora do Laboratório de Pesquisa em Design Visual (LabVisual). É autora de vários artigos e livros sobre tipografia, semiótica e design, e responsável pela plataforma digital Tipografia Paulistana <http://www.fau.usp.br/tipografiapaulistana>. Seus interesses atuais de pesquisa são história da tipografia no Brasil e letras no espaço público.

ORCID < https://orcid.org/0000-0002-2540-770X >. 